\title{
Sugar Cane-Converted Graphene-like Material for the Superhigh Adsorption of Organic Pollutants from Water via Coassembly Mechanisms
}

\author{
Xin Xiao ${ }^{\dagger, \ddagger}$, Baoliang Chen ${ }^{\star},+$, , Lizhong Zhu ${ }^{\dagger, \ddagger}$, and Jerald L. Schnoor ${ }^{\S}$ \\ † Department of Environmental Science, Zhejiang University, Hangzhou 310058, China \\ ‡ Zhejiang Provincial Key Laboratory of Organic Pollution Process and Control, Hangzhou \\ 310058, China \\ $\S$ Department of Civil and Environmental Engineering, University of lowa, lowa City, lowa 52242, \\ United States
}

\begin{abstract}
A sugar cane-converted graphene-like material (FZS900) was fabricated by carbonization and activation. The material exhibited abundant micropores, water-stable turbostratic single-layer graphene nanosheets, and a high BET- $\mathrm{N}_{2}$ surface area $\left(2280 \mathrm{~m}^{2} \mathrm{~g}^{-1}\right)$. The adsorption capacities of FZS900 toward naphthalene, phenanthrene, and 1-naphthol were 615.8, 431.2, and $2040 \mathrm{mg} \mathrm{g}^{-1}$, respectively, which are much higher than those of previously reported materials. The nonpolar aromatic molecules induced the turbostratic graphene nanosheets to agglomerate in an orderly manner, forming 2-11 graphene layer nanoloops, while polar aromatic compounds induced high dispersion or aggregation of the graphene nanosheets. This phase conversion of the nanosized materials after sorption occurred through coassembly of the aromatic molecules and the singlelayer graphene nanosheets via large-area $\pi$ - $\pi$ interactions. An adsorption-induced partition mechanism was further proposed to explain the nanosize effect and nanoscale sorption sites observed. This study indicates that commonly available biomass can be converted to graphene-like material with superhigh sorption ability in order to remove pollutants from the environment via nanosize effects and a coassembly mechanism.
\end{abstract}

\section{Graphical Abstract}

\footnotetext{
This is an open access article published under an ACS AuthorChoice License, which permits copying and redistribution of the article or any adaptations for non-commercial purposes.

*Corresponding Author: Phone: 0086-571-88982587. Fax: 0086-571-88982587. blchen@zju.edu.cn. ASSOCIATED CONTENT

Supporting Information

The Supporting Information is available free of charge on the ACS Publications website at DOI: 10.1021/acs.est.7b03639.

Notes

The authors declare no competing financial interest.
} 


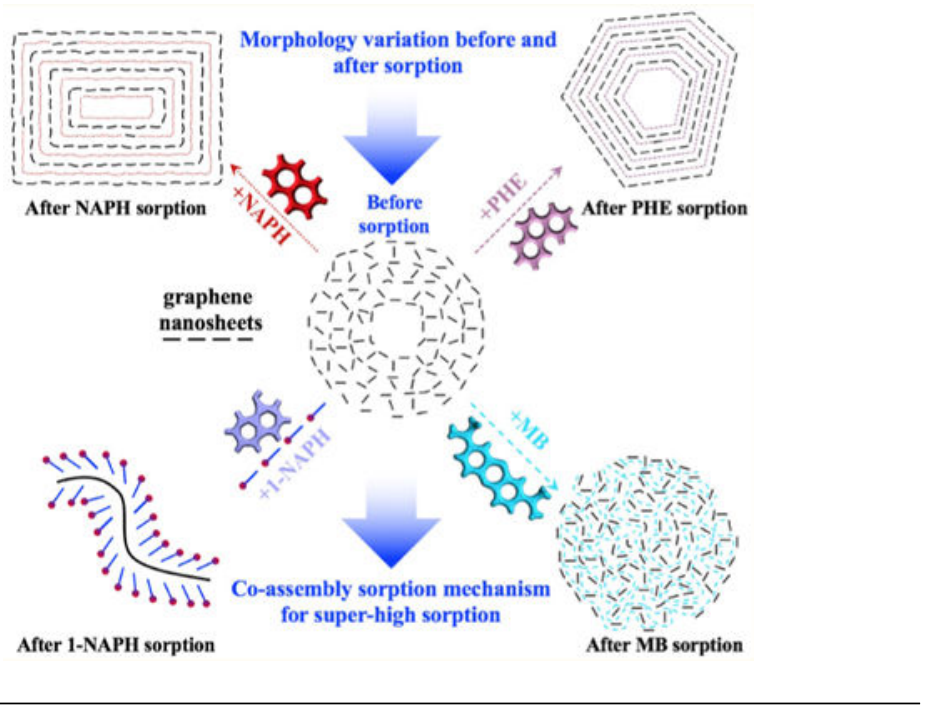

\section{INTRODUCTION}

Thousands of industrial and natural chemical compounds discharge into receiving waters every day. Toxic and persistent organic micropollutants threaten human health, ${ }^{1}$ making cost-effective and appropriate remediation materials and technologies necessary. Sorption is relatively mature, low-cost, and highly efficient technology for pollutant remediation, and researchers continue to seek effective sorbents with higher surface areas, partition coefficients, and sorption capacities. ${ }^{2}$ For traditional carbon-rich sorbents (such as charcoal, soot, and biochar), ${ }^{3,4}$ the condensed structures consume their large sorption potential via limiting internal sites. ${ }^{5}$ Therefore, novel sorbents with extended structures are gaining more and more attention.

Graphene is a novel two-dimensional material made of single-atom-thick carbon with a honeycomb crystal lattice, ${ }^{6,7}$ and it shows great potential for environmental applications. ${ }^{8-10}$ With its high theoretical surface area $\left(2630 \mathrm{~m}^{2} \mathrm{~g}^{-1}\right)$ and strong $\pi-\pi$ interactions, ${ }^{9,11}$ graphene seems to be an ideal material to sorb toxic aromatic pollutants. However, graphene is not economically affordable to manufacture, on large scale even when prepared via the commonly known Hummers method, which also requires large chemical inputs. Chemical vapor deposition (CVD) can produce high-quality graphene, but the process requires large resource and energy input. Biomass sources, such as soybean oil, cookies, chocolate, grass, plastics, roaches, and dog feces, have been studied as precursors for CVD in order to reduce resource consumption. ${ }^{12,13}$ However, large-scale production is still difficult to achieve economically with the CVD method. Moreover, due to strong hydrophobic interactions and large-area $\pi-\pi$ interactions, graphene nanosheets are unstable in water and tend to selfaggregate before associating with pollutant molecules, ${ }^{14}$ which inhibits the adsorption of single-layer graphene nanosheets and then limits their potential applications. Besides graphene, for other novel carbon-rich sorbents (such as fullerene and carbon nanotubes), the self-aggregate interaction consumes lots of sorption sites. Therefore, the development of sorbent with stable expanded structure in the aqueous phase is challenging and necessary for environmental application. 
Biomass is derived from numerous sources and is comparatively cheaper; it could be the agricultural waste and is commonly found in large quantity around the world every year and also requires management and disposal. ${ }^{15}$ For example, in 2005, the worldwide production of sugar cane waste exceeded 1018 million tons. ${ }^{16}$ The conversion of biomass to graphenelike material composed of single-or a few-layer nanosheets has the potential to solve the challenges mentioned above. In this study, an inexpensive and stable graphene-like material was successfully synthesized via the carbonization and activation of sugar cane as a representative biomass source. The preparation of the graphene-like material and possible reaction route are illustrated in Figure 1. Briefly, $\mathrm{FeCl}_{3}$ and $\mathrm{ZnCl}_{2}$ were mixed with sugar cane biomass in a water bath, and then the mixture was carbonized at $900{ }^{\circ} \mathrm{C}$ under $\mathrm{N}_{2}$ atmosphere, during which $\mathrm{a}-\mathrm{Fe}$ and $\mathrm{Zn}$ nanoparticles were generated in situ via reaction with the reduction gas released from the thermal degradation of the sugar cane. ${ }^{17} \mathrm{The} \mathrm{ZnCl}_{2}$ and $\mathrm{Zn}$ nanoparticles evaporated due to the low boiling point of 723 and $906{ }^{\circ} \mathrm{C}$, respectively ${ }^{18}$ (Figure 1). The metal components in the carbonized solid included a-Fe, $\mathrm{Fe}_{3} \mathrm{C}$, and $\mathrm{FeO}(\mathrm{OH})$, based on the characterization of the $\mathrm{X}$-ray diffraction pattern (Figure $\mathrm{S} 1$ ), which functioned as templates for the graphene nanosheets. After the metal residues were removed by $\mathrm{HCl}$, the final carbonized sugar cane materials were obtained and are denoted ${ }_{3}-\mathrm{ZnCl}_{2}$-sugar cane- $900{ }^{\circ} \mathrm{C}$ (abbr. FZS900) (Figure 1). The adsorption capabilities of naphthalene, phenanthrene, 1-naphthol, methylene blue, and toluene by FZS900 were evaluated. The nanostructure of FZS900 before and after sorption was characterized by highresolution transmission electron microscope (HR-TEM) and employed to prove the novel coassembly mechanism.

\section{MATERIALS AND METHODS}

\section{Materials.}

Sugar cane was purchased from a fruit market near the Zijingang Campus, Zhejiang University, Hangzhou, China. The sugar cane was peeled off properly and thoroughly rinsed three times with mill-Q water before use. Analytical grade $\mathrm{FeCl}_{3}$ and $\mathrm{ZnCl}_{2}$ were purchased from Shanghai Chemicals, Shanghai, China. Naphthalene (NAPH > 98\%) and methylene blue ( $\mathrm{MB}>98 \%$ ) were purchased from Acros Organics. Phenanthrene (PHE > 98\%) was supplied by J\&K Chemical. 1-Naphthol (1-NAPH > 99\%) was obtained from Aladdin. Toluene was obtained from Hangzhou Chemical Reagent Co., Ltd., China with analytical grade. All other solvents such as methanol and acetonitrile that were used for high performance liquid chromatography (HPLC) were purchased from Honeywell/Burdick \& Jackson, with HPLC grade. The eluent water was mill-Q water.

\section{FZS900 Preparation.}

The schematic preparation procedures of FZS900 are shown in Figure 1. In general, FZS900 was produced by simultaneous graphitization and activation and with $\mathrm{FeCl}_{3}$ and $\mathrm{ZnCl}_{2}{ }^{19}$ from agricultural biomass waste (sugar cane) which is abundantly available in China. ${ }^{20}$ Briefly, $3.0 \mathrm{~g}$ of sugar cane bulk material was placed into a $100 \mathrm{~mL}$ beaker with $50 \mathrm{~mL}$ of $3.0 \mathrm{~mol} \mathrm{~L}-1 \mathrm{FeCl}_{3}$ aqueous solution. After adding $9.0 \mathrm{~g}$ of $\mathrm{ZnCl}_{2}$, the mixture was placed into a stirred water-bath at $80{ }^{\circ} \mathrm{C}$ for 2 days. At this time, the sugar cane bulk turned to powder form, and $\mathrm{FeCl}_{3}$ and $\mathrm{ZnCl}_{2}$ penetrated inside the sugar cane. Then the mixture was 
moved to a drying oven for $\sim 5$ days at $80{ }^{\circ} \mathrm{C}$ to evaporate water. The solid product was then transferred to a quartz boat and placed into a pipe furnace (GSL-1600X, Hefei Kejing Material Technology Co., Ltd., China) under $\mathrm{N}_{2}$ flow $\left(0.6 \mathrm{~mL} \mathrm{~min}^{-1}\right)$. Before starting the heating program, the air inside the pipe furnace was exhausted by using a vacuum pump prior to heating, and it was refilled with $99.999 \% \mathrm{~N}_{2}$ gas three times. The mixture was heated up to $900{ }^{\circ} \mathrm{C}$ with a controlled heating rate of $5{ }^{\circ} \mathrm{C} \mathrm{min}^{-1}$ and held for $1 \mathrm{~h}$. After naturally cooling down to room temperature, the solid was transferred into $100 \mathrm{~mL}$ of $2 \mathrm{~mol}$ $\mathrm{L}^{-1} \mathrm{HCl}$ aqueous solution and stirred for $8 \mathrm{~h}$ three times. Copious bubbles were generated during the stirring. After rinsing with milli-Qwater 5 times, the residue was vacuum freezedried (LGJ-18A, Beijing Sihuan Instrument Company, China) and passed through a 100mesh sieve ( $<0.154 \mathrm{~mm}$ size). Finally, the graphene-like material was obtained yielding around $14.12 \%$ and referred to as " $\mathrm{FeCl}_{3}-\mathrm{ZnCl}_{2}$-Sugar900" (abbr. FZS900). FZS900 was further characterized and utilized in batch sorption experiments.

\section{Batch Sorption Experiments.}

The methods for the sorption experiments of naphthalene and phenanthrene were cited from previous literature. ${ }^{9,21}$ Detailed methods regarding the sorption kinetics of naphthalene and phenanthrene, the sorption isotherm of naphthalene, phenanthrene, methylene blue, and 1naphthol, and the data analysis of sorption isotherm were given in Supporting Information. Sorption kinetics results indicated that the required sorption equilibrium time of NAPH and PHE onto FZS900 was approximately $5 \mathrm{~min}$ and $24 \mathrm{~h}$, respectively (Figure S2). A stabilized $\mathrm{pH}$ (around $\mathrm{pH}=7.0$ ) for both 1-NAPH and MB isotherm sorption experiments was confirmed (Figure S3) to achieve a stable UV spectra. The unchanged UV spectra of 1NAPH before and after sorption results indicate that there was no transformation of 1-NAPH during the sorption (Figure S4), and the detailed operation conditions for anlyzing NAPH and PHE via HPLC were provided in Tables S1 and S2, respectively.

\section{Sorption Test for Toluene Gas.}

The sorption of toluene, an important volatile organic pollutant, onto FZS900 was also tested. $14.99 \mathrm{mg}$ of FZS900 was weighed on an empty small glass culture Petri dish which was placed on a larger glass culture Petri dish. Then, a $1 \mathrm{~mL}$ toluene droplet was placed on the larger dish as shown in Figure S5. The whole dish was covered with another glass dish for $15 \mathrm{~min}$, and the sorbed quantity of FZS900 was determined by calculating the mass difference before and after sorption. The sorbed quantity of toluene was $8.09 \mathrm{mg}$, yielding a sorption quantity of $539.7 \mathrm{mg} \mathrm{g}^{-1}$ of FZS900.

\section{Theoretical Calculation of Monolayer Sorption Quantity.}

Based on the van der Waals radius, the molecular area of NAPH, PHE, 1-NAPH, and MB is calculated to be $0.732 \mathrm{~nm}^{2}, 1.00 \mathrm{~nm}^{2}, 0.789 \mathrm{~nm}^{2}$, and $1.30 \mathrm{~nm}^{2}$, respectively. ${ }^{22,24}$ Properties of those sorbates are given in Table S3. Then the theoretical estimated sorption values from the maximal monolayer arrangement can be calculated by the following equation

$$
Q_{\mathrm{T}}=\frac{S_{\mathrm{A}} \times M_{\mathrm{r}}}{S_{\mathrm{m}} \times N_{\mathrm{A}}} \times 10^{21}
$$


where $\mathrm{Q}_{\mathrm{T}}$ refers to the theoretical estimated sorption quantity, $\mathrm{mg} \mathrm{g}^{-1} ; \mathrm{S}_{\mathrm{A}}$ is the surface area of FZS900, $\mathrm{m}^{2} \mathrm{~g}^{-1}$, which is $2280 \mathrm{~m}^{2} \mathrm{~g}^{-1}$ (Figure 2); $\mathrm{S}_{\mathrm{m}}$ is the molecular area of NAPH (or PHE, or 1-NAPH, or MB), $\mathrm{nm}^{2} ; \mathrm{N}_{\mathrm{A}}$ is Avogadro's constant, $6.022 \times 10^{23} \mathrm{~mol}^{-1}$; and $\mathrm{M}_{\mathrm{r}}$ is the molecular weight of NAPH (or PHE), $\mathrm{g} \mathrm{mol}^{-1}$. The theoretical calculated monolayer sorption quantities of NAPH, PHE, 1-NAPH, and MB onto FZS900 are $663.3 \mathrm{mg} \mathrm{g}^{-1}, 674.9$ $\mathrm{mg} \mathrm{g}^{-1}, 692.2 \mathrm{mg} \mathrm{g}^{-1}$, and $931.9 \mathrm{mg} \mathrm{g}^{-1}$, respectively. Considering the highest sorption quantities of FZS900 to NAPH, PHE, 1-NAPH, and MB are $615.9 \mathrm{mg} \mathrm{g}^{-1}, 431.2 \mathrm{mg} \mathrm{g}^{-1}$, $2040 \mathrm{mg} \mathrm{g}^{-1}$, and $474.7 \mathrm{mg} \mathrm{g}^{-1}$, then 1-NAPH seems to be the only compound that shows multilayer sorption behavior onto FZS900 in this work, which is consistent with a previous report. ${ }^{23} \mathrm{~A}$ comparison of the adsorption data of FZS900 in the current study with that of traditional and novel materials from the literature was provided in Figure 3.

\section{Structural Characterization.}

BET-N $\mathbf{N}_{2}$ - The surface area and pore size distribution of FZS900 were analyzed by the Brunauer-Emmett-Teller method (BET-N ${ }_{2}$ ) on a NOVA- 2000E surface area analyzer using $\mathrm{N}_{2}$ as the gas molecule $\left(0.162 \mathrm{~nm}^{2}\right)$. In brief, $17.47 \mathrm{mg}$ of FZS900 was degassed under vacuum (to less than $0.1 \mathrm{~Pa}$ ) for $12 \mathrm{~h}$ at $378 \mathrm{~K}$ After it cooled down to room temperature naturally, the $\mathrm{N}_{2}$ sorptiondesorption analysis started under liquid $\mathrm{N}_{2}$ temperature $77 \mathrm{~K}$. Surface area was determined by the Brunauer-Emmett-Teller (BET) method, using seven data sorption points, between relative pressures of 0.05 and 0.3 based on the monolayer adsorption theory. Pore distribution was calculated by all the desorption data points according to the Barrett-Joyner-Halenda (BJH) method. Data are presented in Figure 2 (A, E).

SEM.-A scanning electron microscope (SEM) (FEI Quanta 3D FEG) was utilized to observe the surface morphology of FZS900. A tiny sample was directly adhered to the double-coated carbon-conductive tabs on stubs. Then the stub was gilded with Pt for $60 \mathrm{~s}$ to enhance the sample conductivity prior to SEM observation under $5 \mathrm{kV}$. Data are presented in Figure 2 (B, F).

AFM.-Atomic force microscope (AFM) images of FZS900 on cleaved mica were taken under a tapping mode by atomic force microscopy (AFM, SPI-3800N, Seiko Co.) After sonicating for $30 \mathrm{~min}$, a droplet of FZS900 dispersion $\sim 0.01 \mathrm{mg} / \mathrm{mLwas}$ cast onto the fresh mica surface. The sample was then kept under an infrared lamp at room temperature to allow the water to evaporate before the AFM investigation. Results are shown in Figure S6.

TEM.-Morphologies at the nanoscale and atomic scale of FZS900, FZS900+NAPH, and FZS900+PHE were observed by applying high-resolution transmission electron microscopy (HR-TEM). In order to observe the nanosheet structure of FZS900, a low boiling point solvent, chloroform was used to exfoliate the nanosheet graphene in FZS900 according to previous reports ${ }^{25,26}$ because a low boiling point solvent can be easily removed before the sample enters the vacuum system of TEM. To be more specific, $4.0 \mathrm{mg}$ of the FZS900 sample was added into a $40 \mathrm{~mL}$ glass bottle with $40 \mathrm{~mL}$ of trichloro-methane (AR, Sinopharm Chemical Reagent co., Ltd., China). The suspension was further sonicated (SK7200HP, Shanghai Kedao Ultrasonic Instrument Co, Shanghai, China) at 40\% power (53 
$\mathrm{kHz}$, power: $350 \mathrm{~W}$ ) for $30 \mathrm{~min}$. After centrifuging at $500 \mathrm{rpm}$ for $90 \mathrm{~min}$, the supernatants were dropped into carbon-film-coated copper microgrid. Since $\mathrm{CHCl}_{3}$ is a low boiling point solvent, it dried fast in several minutes before observations with HR-TEM (FEI Tecnai G ${ }^{2}$ F20 S-TWIN) operated at $200 \mathrm{kV}$ attached with energy X-ray dispersive spectrometry (EDS) to achieve the element distribution of FZS900. The corresponding images are shown in Figure 2(C, D, G, H), and the corresponding EDS results were given in Figure S7. For the morphology observation of FZS900 before sorption, the original FZS900 sample was tested by directly putting the sample (dispersed in water) onto the carbon-film-coated copper microgrid, and for FZS900 after sorption (including NAPH, PHE, 1-NAPH, and MB sorption), the highest-sorbate-concentration mixture in the isotherm experiments was dipped onto carbon-film-coated copper microgrids in order to achieve the morphology of FZS900 with the highest sorption quantity in our experiments. HR-TEM (FEI Tecnai G ${ }^{2}$ F20 STWIN) operated at $200 \mathrm{kV}$ was applied for FZS900 morphology variation after NAPH, PHE, 1-NAPH, and MB sorption, and the corresponding images were shown in Figure 4(A, C, G, I) and Figure S2(A, B, C, D, F, G, H). EDS spectrum of FZS900+PHE was also recorded to obtain the element distribution and was provided in Figure S7. To achieve better results, HR-TEM equipped with a Cs-corrector (FEI Titan 80-300) was also operated at 80 $\mathrm{kV}$ for the observation of FZS900 sorbed with PHE. Corresponding results are presented in Figure 4(E) and Figure S2(E).

XRD.-X-ray powder diffraction (XRD) was applied to monitor the mineral phase of FZS900 before $\mathrm{HCl}$ washing. The sample directly used the carbonized solid after pyrolysis of $\mathrm{FeCl}_{3}, \mathrm{ZnCl}_{2}$, and sugar cane. The spectrum was obtained at a diffraction angle region of $5-70^{\circ}$ by an X-ray diffractometer (RIGAKU D/MAX 2550/PC, Japan) with $\mathrm{Cu}-\mathrm{Ka}$ radiation. The resulting spectrum is shown in Figure S1.

XPS.-X-ray photoelectron spectroscopy (XPS) is a powerful and sensitive technology to measure the surface elemental compositions of solid samples. The XPS characterization was performed via a VG Escalab Mark II with a scanning step of $0.5 \mathrm{eV}$ from 0 to $1000 \mathrm{eV}$. The results were shown in Figure S8. The C 1s spectra of FZS900 and FZS900+PHE samples were recorded at $284.4 \mathrm{eV}$ with a scanning step of $0.2 \mathrm{eV}$ as shown in Figure S9.

Raman.-Raman spectra of FZS900, FZS900+PHE, and FZS900 + 1-NAPH were characterized to monitor the transformation of the graphene-like nanosheet layers by a Lab Ram HRUV Raman spectrometer (JDbin-yvon, FR). The laser excitation wavelength was $514 \mathrm{~nm}$, and the Raman spectra was measured from 100 to $3500 \mathrm{~cm}^{-1}$ with a step of 0.444 $\mathrm{cm}^{-1}$. The FZS900+PHE and FZS900-1-NAPH samples for the Raman test were prepared by vacuum filtrating the highest- sorbate-concentration mixture from the isotherm experiments with a porous alumina membrane (pore size: $200 \mathrm{~nm}$, Whatman). The membrane with FZS900+PHE and FZS900 + 1-NAPH was further oven-dried for Raman characterization. Results are shown in Figure S9.

FTIR.-Fourier transform infrared spectroscopy (FTIR) was utilized to record the functional groups of FZS900 before and after 1-NAPH and MB sorption. Briefly, after freeze-drying, the samples was mixed with $\mathrm{KBr}$ (at a ratio of $0.5 \mathrm{mg}$ sample/100 $\mathrm{mg} \mathrm{KBr}$ ), ground, and 
pressed. Then the FTIR spectra was recorded using a Nicolet 6700 spectrometer in the 400 $4000 \mathrm{~cm}^{-1}$ region with resolution of $1 \mathrm{~cm}^{-1}$. For all the samples, the air background spectrum was subtracted, and 64 interferograms were collected. The corresponding spectra are shown in Figure S10.

\section{RESULTS AND DISCUSSION}

\section{Morphology of FZS900.}

The surface and structural morphology of FZS900 was observed by different characterization techniques. $\mathrm{N}_{2}$ sorption-desorption results at $1 \mathrm{~atm}$ and $77 \mathrm{~K}$ using the Brunauer-Emmett-Teller method (BET- $\left.\mathrm{N}_{2}\right)$ show that FZS900 has a sorption capacity of $2866 \mathrm{mg} \mathrm{g}^{-1}$ for $\mathrm{N}_{2}$ (Figure $2 \mathrm{~A}$ ), which is much higher than that reported for other metalorganic frameworks. ${ }^{27}$ The calculated surface area of FZS900 $\left(2280 \mathrm{~m}^{2} \mathrm{~g}^{-1}\right)$ is close to the theoretical surface area of single-layer graphene nanosheets $\left(2630 \mathrm{~m}^{2} \mathrm{~g}^{-1}\right)$, implying a less agglomerated graphene nanosheet structure. The pore size distribution (Figure 2E, red line) indicated that abundant mesopores $(2-50 \mathrm{~nm})$ were present in FZS900, which was also observed in the SEM images (Figure 2B and 2F). In addition, the AFM images of FZS900 show that the graphene nanosheets have a depth of $\sim 1.2 \mathrm{~nm}$ (Figure S6). Although the pure single layer graphene has a thickness of $0.34 \mathrm{~nm}$, it is widely believed that a depth around 1 $\mathrm{nm}$ could be seen as a single-layered structure due to the existence of functional groups, structural defects, and adsorbed water molecules. ${ }^{28,29}$ Also, the high-resolution transmission electron microscopy (HR-TEM) images demonstrated the microporous $(<2 \mathrm{~nm})$ structure of FZS900 (Figure 2C and 2G), as well as some atomically resolved graphene structures (Figure 2D and2H), considering that the X-ray photoelectron spectroscopy (XPS) (Figure S8) results indicated that carbon was the major component. The abundant microspores in FZS900 could induce the formation of a square ice-like structure for water confined in the micropores, which was experimentally confirmed by Algara-Siller et al. ${ }^{30}$ and which may help to stabilize the graphene-like structure of FZS900 by avoiding self-agglomeration in water. The stability of FZS900 was also suggested by the high sorption capacity of FZS900, which will be discussed below.

\section{Superhigh Adsorption Capability of FZS900 toward Organic Pollutants.}

Because the graphene nanosheets were stable in water, FZS900 could exert its full potential adsorption capability toward typical toxic organic pollutants due to its high surface area. The adsorption capacity of FZS900 for NAPH, PHE, 1-NAPH, and MB was 615.8, 431.2, 2040, and $474.7 \mathrm{mg} \mathrm{g}^{-1}$ for the sorbate equilibrium concentration $\left(\mathrm{C}_{\mathrm{e}}\right)$ as $16.44,0.51,103.4$, and $128.4 \mathrm{mg} \mathrm{L}^{-1}$. To the best of our knowledge, this adsorption capacity of FZS900 for NAPH, PHE, and 1-NAPH is comparatively higher than any other novel or traditional sorbents as reported earlier. Considering the same aqueous concentration for NAPH, PHE, and 1-NAPH (Figure 3), the adsorption capacities of FZS900 toward NAPH, PHE, and 1-NAPH were orders of magnitude higher than those of other reported carbon materials. For example, the adsorption capacity of FZS900 for NAPH was approximately three times greater than that of pine needle-derived biochar at $700{ }^{\circ} \mathrm{C}^{3}$ and graphene made from the Hummers method, ${ }^{9}$ two times higher than that of commercial activated carbon ${ }^{3}$ and sulfonated graphene, ${ }^{10} 70$ times higher than that of multiwalled carbon nanotubes, ${ }^{31}$ and almost 200 times higher than that of 
graphene oxide. ${ }^{9}$ In addition, the adsorption capacity of FZS900 for toluene gas, an important aromatic air pollutant, could be directly weighed by a microbalance and was found to be $539.7 \mathrm{mg} \mathrm{g}^{-1}$ (Figure S5).

\section{Coassembly Sorption Mechanisms.}

The superhigh adsorption of FZS900 results from its stable single-layer graphene nanosheet structure, in which each graphene nanosheet is vertically offset, as shown in the HRTEM images in Figure 4A. Spherical turbostratic nanosheet structures in the original FZS900 sample were observed and are schematically illustrated in Figure 4B. Interestingly, following the sorption of polycyclic aromatic hydrocarbons (PAHs), such as NAPH and PHE, the spherical turbostratic nanosheet structures were converted into multiple nanoloops made up of 2-11 graphene layers, as shown in the HRTEM images in Figure 4C for NAPH and Figure 4E for PHE. These are the first observations of this type of structure. To confirm the PAH-induced morphology transformation of FZS900, three instrumental methods were utilized to characterize FZS900 with and without PHE sorption: 1) energy dispersive spectroscopy (EDS); 2) C 1s X-ray photoelectron spectroscopy; and 3) Raman spectroscopy. The EDS spectra (Figure S7) indicated that carbon was the major constituent, and the increase in the $\mathrm{C}-\mathrm{C} / \mathrm{C}=\mathrm{C}$ ratio of the total carbon content (Figure S9) confirmed that PHE adsorption correlated with the overall $\mathrm{C}-\mathrm{C} / \mathrm{C}=\mathrm{C}$ ratio in the $\mathrm{PHE}$ molecule. In addition, the 2D band disappeared after sorption as observed in Raman spectra (Figure S9), and Raman intensity ratios of $\mathrm{D}$ band to $\mathrm{G}$ band decreased once the number of stacking layers accumulated. ${ }^{32}$ The agreement between the Raman results and the HRTEM images suggests that a more ordered structure was formed after NAPH and PHE adsorption. The interlayer spacing between stacked graphene nanosheets was $0.3457 \pm 0.0031 \mathrm{~nm}$ and $0.3447 \pm 0.0013$ $\mathrm{nm}$ after NAPH and PHE sorption, respectively (Figure 4C and 4E), which is slightly larger than the graphene distance of $0.335 \mathrm{~nm}$ in graphite, suggesting that NAPH and PHE were intercalated into the interlamellar region of the graphene nanosheets. Therefore, the small molecule-induced self-assembly of graphene nanosheets is hypothesized to explain the interaction mechanism between NAPH (or PHE) and graphene nanosheets via $\pi-\pi$ interactions, as shown in Figure 4D for NAPH and Figure 4F for PHE. It is well-known that the aromatic surfaces could not be exposed fully due to agglomeration in traditional sorbents (such as soot and biochar), while in the case of FZS900, aromatic sheets were exposed fully thus offering self-assembly for NAPH and PHE molecules. In fact, the shape of the nanoloops is controlled by the molecular structures of the PAHs to some extent. For instance, the nanoloop is nearly quadrilateral and hexagonal after NAPH and PHE (Figure 4C) association as explained by smaller molecular size producing larger angles. Therefore, the adsorption of PAHs onto FZS900 occurs through coassembly of the aromatic molecules and the single-layer graphene nanosheets. The coassembly mechanism is recognized as a convincing new mechanism for materials having superhigh sorption capacity.

Generally, the prepared graphene nanosheets were not perfect and instead contained wrinkles, flat aromatic surfaces, defects, and oxygen-containing functional groups. ${ }^{33}$ The $\pi$ electron system in PAHs leads to strong $\pi-\pi$ interaction forces between PAH molecules and the aromatic surface of the graphene nanosheets. ${ }^{34}$ These $\pi-\pi$ interactions cause the microporous graphene nanosheets to form more ordered, layer-to-layer, looplike structures 
in order to generate more $\pi-\pi$ interactions between the graphene nanosheets, thereby reducing the energy of the system. Also, the spherical turbostratic nanosheets of FZS900 contained many mesopores and micropores. When PAH molecules are present, they replace the crystalline ice-like water molecules in the confined micropores space ${ }^{30}$ of FZS900, and they generate large-area $\pi-\pi$ interactions due to the hydrophobicity of the PAHs, resulting in the destruction of the original ice-like water structure. Originally, the ice-like water structure helped to stabilize the meso-and microporous structures in FZS900. Replacement of water molecules leads to the collapse of the porous structures and a coassembly ordering process, resulting in the formation of 2-11 layer loops. Although the ordering of graphene nanosheets is an entropy-decreasing process, the replacement of the ordered ice-like water molecules increases the entropy, thereby preserving the overall environmental entropy.

However, the ice-like water in graphene pores was not fully replaced when 1-NAPH and MB were present due to their hydrophilic functional groups, and thus the self-assembly of graphene nanosheets did not occur, as shown in Figure 4G and Figure 4I. In fact, 1-NAPH enhanced the dispersion of graphene nanosheets in FZS900, as shown in Figure 4G, and a surfactant-like effect was observed, as in Figure S9. Fluorescence occurred under the 514 $\mathrm{nm}$ laser excitation used for Raman characterization (Figure S9), which may be due to some of the nanosized graphene sheets being dispersed upon sorption of 1-NAPH. In contrast, the graphene nanosheets generally aggregated after MB sorption (Figure 4I).

Overall, we believe that the PAH-induced the process of $\pi-\pi$ interaction and self-assembly unfolded the wrinkles and folds of the graphene nanosheets, which are considered to be fast and efficient adsorption sites for PAHs. ${ }^{34}$ This type of self-assembly process could precede a superhigh sorption ability for aromatic pollutants, with simultaneous entropic ordering, ${ }^{35,36}$ making the process a coassembly process. Since the $\pi-\pi$ interactions are only one kind of nanoscale force occurring during the self-assembly process, other nanoscale forces may be utilized for selective pollutant removal and recovery in the future. ${ }^{35,37,38}$ The nanosized graphene nanosheets in FZS900 (Figure 2D and 2H and Figure S2) contributed to the PAHinduced coassembly process - a nanoeffect, since larger-sized graphene sheets cannot unfold themselves easily due to steric effects. In addition, NAPH being the smaller size molecule could lead to a more fluctuated self-assembly structure (Figure 4C and 4D) compared to PHE (Figure 4E and 4F). The theory based on traditional adsorption of carbon sorbents, such as activated carbon, biochar, ${ }^{3,39,40}$ soot, ${ }^{41}$ and soil organic matter, ${ }^{42}$ generally contains invariable adsorption sites and static morphology. In the current study, the ordering selfassembly adsorption mechanism is proposed for the first time. This mechanism provides new insight into addressing pollutants in wastewater by taking advantage of the coassembly adsorption process, which enhances selectivity for pollutant removal and recovery. Furthermore, the traditional adsorption mechanisms require the renewal of the sorbent material, especially for superhigh sorption quantities, which could cause structural rearrangement of sorbent, as was recently reported for gas sorption-induced structural deformation and pore contraction of metal-organic frameworks, MOFs. ${ }^{43}$ 


\section{The Sorption Isotherms of FZS900.}

The sorption isotherms of NAPH, PHE, 1-NAPH, and MB were examined experimentally to analyze the sorption performance of FZS900 under different $\mathrm{C}_{\mathrm{e}}$ (Figure 5). The fast sorption kinetics (Figure S11) of NAPH and PHE incriminates the presence of strong forces between the PAHs and graphene nanosheets. At low PAH concentrations, the partition coefficient value $\left(\mathrm{K}_{\mathrm{d}}, \mathrm{L} \mathrm{kg}^{-1}\right)$ of NAPH and PHE reaches $2 \times 10^{6}$ and $2 \times 10^{7}$ (Figure S12), respectively, demonstrating the potential removal for trace pollutants from contaminated waters. As the aqueous concentration of the PAHs increased, the sorption isotherms of the PAHs showed a linear partition-like sorption mechanism, similar to a liquidation-like process. In other words, the PAH molecules replaced the water microdroplets near the aromatic defects and wrinkles and micropores of the graphene nanosheets. ${ }^{44}$ The sorption of PAHs from the water phase to the solid phase is similar to a gas-liquidation process, resulting in a large amount of PAHs sorbed and leading toward an enthalpic-and entropicdriven ordering process, which is in accordance with current HRTEM results. 1-NAPH and MB were also monitored as typical oxygen-containing and dye pollutants, respectively, and the FTIR spectra offered substantial evidence for the strong sorption of 1-NAPH and MB to FZS900 (Figure S10).

A steady sorbate concentration before and after sorption (Figure S13) implies negligible loss from environmental factors, such as volatilization or sorption by the glass bottle. The sorption quantity sorbed of 1-NAPH was greater than that of the mass of sorbent itself, $\sim 2000 \mathrm{mg} \mathrm{g}^{-1}$ under high aqueous equilibrium concentrations. The percent removal of MB from the water phase reached $99.2 \%$ at an initial MB concentration of $10 \mathrm{mg} \mathrm{L}^{-1}$, as shown in Figure 5D. The sorption capacity of MB was $474.7 \mathrm{mg} \mathrm{g}^{-1}$. Toluene, an important air pollutant, adsorbed onto FZS900 at a quantity of $539.7 \mathrm{mg} \mathrm{g}^{-1}$. These results indicate the great potential of FZS900 for environmental containment and water purification. In addition, a possible recycling process in the preparation of FZS900 is proposed in Figure 1 to reduce the chemical consumption and potential environmental impact when preparing FZS900. Unlike graphene preparation or $\mathrm{KOH}$ activation processes, the consumed chemicals, such as $\mathrm{HCl}, \mathrm{FeCl}_{3}$, and $\mathrm{ZnCl}_{2}$, could be recycled, and some flammable gases $\left(\mathrm{CH}_{4}, \mathrm{CO}\right.$, and $\left.\mathrm{H}_{2}\right)$ could be collected during the preparation process to recover energy, which makes the already inexpensive sugar cane precursors of FZS900 more economically affordable.

For a sorbent applied for different initial aqueous concentrations $\left(C_{0}\right)$, here we consider "capability" as the lowest $C_{\mathrm{e}}$ value that the sorbent could achieve given a relatively low initial aqueous pollutant concentration, and the word "capacity" refers to the maximum sorbed amount that the sorbent could achieve if the pollutant concentration keeps going higher. Traditionally, partition sorption mechanisms show high capacity at high sorbate concentration but low capability (sorption amount at low $\mathrm{C}_{\mathrm{e}}$ ). In contrast, adsorption shows high capability but low sorption capacity after reaching its maximum adsorption amount. Considering both the sorption ability and capacity when developing sorbents is challenging. In 1979, Chiou et al. proposed a linear sorption isotherm of nonionic organic compounds onto soil organic matter. ${ }^{42}$ However, observing and explaining why these compounds show linear partition sorption behavior onto solid soil organic matter are difficult. Based on the coassembly phenomenon in this work, we suggest an adsorption-induced partition sorption 
mechanism in which the sorbate is adsorbed onto the nanosized sorbent medium by adsorption, causing the nanosized materials to become soft matter during partition sorption. For understanding the adsorption-induced partition sorption mechanism, the definition of partition and adsorption needs to be noticed. A well accepted concept is that partition is a process in which the sorbate penetrated into the lattice structures of the sorbent, and the adsorption is a process in which the sorbate attached on the surface of the sorbent lattice. For the graphite, the interlayers of graphene are part of its lattice, and the pollutants could hardly penetrate into the graphite interlayers, leading to an adsorption mechanism. However, for the graphene nanosheets, the layer-to-layer interaction between the graphene is no longer part of the graphene lattice, instead, the lattice of graphene is the honeycomb-like 2D structures.

That is the reason why we call the graphene-like FZS900 a partition sorption. On the other side, the surface of graphene sorbed a large number of PAHs and further combined with other graphene nanosheets to enlarge its sorption, resulting in a partition phenomenon similar to the PAHs penetrated into the interlayers of graphite, which is also called sorbent softening. Therefore, we proposed an adsorption induced partition sorption mechanism.

This work describes an easily prepared, environmentally friendly, low-cost, and highly efficient material from sugar cane with a stable single-layer graphene nanosheet structure. This graphene-like material has superhigh adsorption capability which can be utilized in wastewater treatment process. ${ }^{45}$ The turbostratic graphene nanosheets in FZS900 were stable in water containing polar molecules, which contribute to the superhigh adsorption of 1-naphthol and MB. Meanwhile, the turbostratic graphene nanosheets are transformed to ordered nanoloops after interaction with naphthalene and phenan-threne, as nonpolar aromatic molecules, which induce the coassembly of PAH molecules (small aromatic units) and graphene nanosheets (large aromatic layers). FZS900 was prepared directly from sugar cane and overcomes the economical and self-aggregation limitations of graphene for environmental applications. The coassembly mechanism initiating from the turbostratic graphene nanosheet structure makes FZS900 one of the best novel functional sorbents for pollutant abatement. This mechanism endows FZS900's extraordinarily enriching ability from the aqueous phase to the solid phase. Presumably, other new materials could be prepared with aromatic organic layers (with $\pi-\pi$ bonding) coassembled with biomassderived graphene-like sheets to make another new and novel sorbent material. More applications via applying small molecules induced coassembled graphene are needed in the future.

\section{Supplementary Material}

Refer to Web version on PubMed Central for supplementary material.

\section{ACKNOWLEDGMENTS}

This project was supported by the National Natural Science Foundations of China (Grant 21425730, 21537005, and 21621005), the National Basic Research Program of China (Grant 2014CB441106), and the National Key Technology Support Program of China (Grant 2015BAC02B01). 


\section{REFERENCES}

(1). Schwarzenbach RP; Escher BI; Fenner K; Hofstetter TB; Johnson CA; von Gunten U; Wehrli B The challenge of micropollutants in aquatic systems. Science 2006, 313 (5790), 1072-1077. [PubMed: 16931750]

(2). Teixido M; Hurtado C; Pignatello JJ; Beltran JL; Granados M; Peccia J Predicting contaminant adsorption in black carbon (biochar)-amended soil for the veterinary antimicrobial sulfamethazine. Environ. Sci Technol 2013, 47 (12), 6197-6205. [PubMed: 23713747]

(3). Chen B; Zhou D; Zhu L Transitional adsorption and partition of nonpolar and polar aromatic contaminants by biochars of pine needles with different pyrolytic temperatures. Environ. Sci. Technol. 2008, 42 (14), 5137-5143. [PubMed: 18754360]

(4). Cao X; Ma LQ; Gao B; Harris W Dairy-manure derived biochar effectively sorbs lead and atrazine. Environ. Sci. Technol. 2009, (9), 3285-3291. [PubMed: 19534148]

(5). Xiao X; Chen B A direct observation of the fine aromatic clusters and molecular structures of biochars. Environ. Sci. Technol. 2017, 51 (10), 5473-5482. [PubMed: 28399630]

(6). Novoselov KS; Geim AK; Morozov SV; Jiang D; Zhang Y; Dubonos SV; Grigorieva IV; Firsov AA Electric field effect in atomically thin carbon films. Science 2004, 306 (5696), 666-669. [PubMed: 15499015]

(7). Stolyarova E; Rim KT; Ryu SM; Maultzsch J; Kim P; Brus LE; Heinz TF; Hybertsen MS; Flynn GW High-resolution scanning tunneling microscopy imaging of mesoscopic graphene sheets on an insulating surface. Proc. Natl. Acad. Sci. U. S. A. 2007, 104 (22), 9209-9212. [PubMed: 17517635]

(8). Kemp KC; Seema H; Saleh M; Le NH; Mahesh K; Chandra V; Kim KS Environmental applications using graphene composites: water remediation and gas adsorption. Nanoscale 2013, 5 (8), 3149-3171. [PubMed: 23487161]

(9). Wang J; Chen Z; Chen B Adsorption of polycyclic aromatic hydrocarbons by graphene and graphene oxide nanosheets. Environ. Sci. Technol. 2014, 48 (9), 4817-4825. [PubMed: 24678934]

(10). Zhao G; Jiang L; He Y; Li J; Dong H; Wang X; Hu W Sulfonated graphene for persistent aromatic pollutant management. Adv. Mater. 2011, 23 (34), 3959-3963. [PubMed: 21800380]

(11). Zhu Y; Murali S; Cai W; Li X; Suk JW; Potts JR; Ruoff RS Graphene and graphene oxide: synthesis, properties, and applications. Adv. Mater. 2010, 22 (35), 3906-3924. [PubMed: 20706983]

(12). Seo DH; Pineda S; Fang J; Gozukara Y; Yick S; Bendavid A; Lam SKH; Murdock AT; Murphy AB; Han ZJ; Ostrikov K Single-step ambient-air synthesis of graphene from renewable precursors as electrochemical genosensor. Nat. Commun. 2017, 8, 14217. [PubMed: 28134336]

(13). Ruan G; Sun Z; Peng Z; Tour JM Growth of graphene from food, insects, and waste. ACS Nano 2011, 5 (9), 7601-7607. [PubMed: 21800842]

(14). Yang K; Wang J; Chen B Facile fabrication of stable monolayer and few-layer graphene nanosheets as superior sorbents for persistent aromatic pollutant management in water. J. Mater. Chem. A 2014, 2 (43), 18219-18224.

(15). Mendu V; Shearin T; Campbell JE; Stork J; Jae J; Crocker M; Huber G; DeBolt S Global bioenergy potential from high-lignin agricultural residue. Proc. Natl. Acad. Sci. U. S. A. 2012, 109 (10), 4014-4019. [PubMed: 22355123]

(16). Purohit P; Michaelowa A CDM potential of bagasse cogeneration in India. Energy Policy 2007, 35 (10), 4779-4798.

(17). Moezzi A; Cortie M; McDonagh A Transformation of zinc hydroxide chloride monohydrate to crystalline zinc oxide. Dalton Transactions 2016, 45 (17), 7385-7390. [PubMed: 27030646]

(18). Dean JA Lange's handbook of chemistry (Section 3), 15th ed.; McGraw-Hill: 1998; p 3.59.

(19). Sun L; Tian C; Li M; Meng X; Wang L; Wang R; Yin J; Fu H From coconut shell to porous graphene-like nanosheets for high-power supercapacitors. J. Mater. Chem. A 2013, 1 (21), 6462 6470.

(20). Chen R; Yuan Z Sugarcane production and research in China. Int. Sugar J. 2010, 112 (1340), 452. 
(21). Chen Z; Chen B; Zhou D; Chen W Bisolute sorption and thermodynamic behavior of organic pollutants to biomass-derived biochars at two pyrolytic temperatures. Environ. Sci. Technol. 2012, 46 (22), 12476-12483. [PubMed: 23121559]

(22). Chen Z; Chen B; Zhou D Composition and sorption properties of rice-straw derived biochars. Acta Scientiae Circumstantiae 2013, 33 (1), 9-19.

(23). Chen B; Chen Z Sorption of naphthalene and 1-naphthol by biochars of orange peels with different pyrolytic temperatures. Chemosphere 2009, 76 (1), 127-133. [PubMed: 19282020]

(24). Hang PT; Brindley G Methylene blue absorption by clay minerals. Determination of surface areas and cation exchange capacities (clay-organic studies XVIII). Clays Clay Miner. 1970, 18 (4), 203-212.

(25). O’Neill A; Khan U; Nirmalraj PN; Boland J; Coleman JN Graphene dispersion and exfoliation in low boiling point solvents. J. Phys. Chem. C 2011, 115 (13), 5422-5428.

(26). Hernandez Y; Lotya M; Rickard D; Bergin SD; Coleman JN Measurement of multicomponent solubility parameters for graphene facilitates solvent discovery. Langmuir 2010, 26 (5), 32083213. [PubMed: 19883090]

(27). Chae HK; Siberio-Perez DY; Kim J; Go Y; Eddaoudi M; Matzger AJ; O’Keeffe M; Yaghi OM A route to high surface area, porosity and inclusion of large molecules in crystals. Nature 2004, 427 (6974), 523-527. [PubMed: 14765190]

(28). Stankovich S; Dikin DA; Piner RD; Kohlhaas KA; Kleinhammes A; Jia Y; Wu Y; Nguyen ST; Ruoff RS Synthesis of graphene-based nanosheets via chemical reduction of exfoliated graphite oxide. Carbon 2007, 45 (7), 1558-1565.

(29). McAllister MJ; Li JL; Adamson DH; Schniepp HC; Abdala AA; Liu J; Herrera-Alonso M; Milius DL; Car R; Prud'homme RK; Aksay IA Single sheet functionalized graphene by oxidation and thermal expansion of graphite. Chem. Mater. 2007, 19 (18), 4396-4404.

(30). Algara-Siller G; Lehtinen O; Wang FC; Nair RR; Kaiser U; Wu HA; Geim AK; Grigorieva IV Square ice in graphene nanocapillaries. Nature 2015, 519 (7544), 443-445. [PubMed: 25810206]

(31). Yang K; Wang X; Zhu L; Xing B Competitive sorption of pyrene, phenanthrene, and naphthalene on multiwalled carbon nanotubes. Environ. Sci. Technol. 2006, 40 (18), 5804-5810. [PubMed: 17007144]

(32). Malard LM; Pimenta MA; Dresselhaus G; Dresselhaus MS Raman spectroscopy in graphene. Phys. Rep. 2009, 473 (5-6), 51-87.

(33). Perreault F; de Faria AF; Elimelech M Environmental applications of graphene-based nanomaterials. Chem. Soc. Rev. 2015, 44 (16), 5861-5896. [PubMed: 25812036]

(34). Wang J; Chen B; Xing B Wrinkles and folds of activated graphene nanosheets as fast and efficient adsorptive sites for hydrophobic organic contaminants. Environ. Sci. Technol. 2016, 50 (7), 3798-3808. [PubMed: 26938576]

(35). Bishop KJM; Wilmer CE; Soh S; Grzybowski BA Nanoscale forces and their uses in selfassembly. Small 2009, 5 (14), 1600-1630. [PubMed: 19517482]

(36). Mann S Self-assembly and transformation of hybrid nano-objects and nanostructures under equilibrium and non-equilibrium conditions. Nat. Mater. 2009, 8 (10), 781-792. [PubMed: 19734883]

(37). Jin J; Iyoda T; Cao C; Song Y; Jiang L; Li T; Zhu D Self-assembly of uniform spherical aggregates of magnetic nanoparticles through pi-pi interactions. Angew. Chem., Int. Ed. 2001, 40 (11), 2135-2138.

(38). Zang L; Che Y; Moore JS One-dimensional self-assembly of planar pi-conjugated molecules: Adaptable building blocks for organic nanodevices. Acc. Chem. Res. 2008, 41 (12), 1596-1608. [PubMed: 18616298]

(39). Xiao X; Chen Z; Chen B H/C atomic ratio as a smart linkage between pyrolytic temperatures, aromatic clusters and sorption properties of biochars derived from diverse precursory materials. Sci. Rep. 2016, 6, 22644. [PubMed: 26940984]

(40). Xiao X; Chen B Interaction mechanisms between biochar and organic pollutants. In Agricultural and Environmental Applications of Biochar: Advances and Barriers; 2016; DOI: 10.2136/ sssaspec-pub63.2014.0044 
(41). Chen B; Huang W Effects of compositional heterogeneity and nanoporosity of raw and treated biomass-generated soot on adsorption and absorption of organic contaminants. Environ. Pollut. 2011, 159 (2), 550-556. [PubMed: 21071121]

(42). Chiou CT; Peters LJ; Freed VH Physical concept of soil-water equilibria for nonionic organic compounds. Science 1979, 206 (4420), 831-832. [PubMed: 17820762]

(43). Krause S; Bon V; Senkovska I; Stoeck U; Wallacher D; Tobbens DM; Zander S; Pillai RS; Maurin G; Coudert F. o.-X.; Kaskel S pressure-amplifying framework material with negative gas adsorption transitions. Nature 2016, 532 (7599), 348-352. [PubMed: 27049950]

(44). Yang K; Chen B; Zhu L Graphene-coated materials using silica particles as a framework for highly efficient removal of aromatic pollutants in water. Sci. Rep. 2015, 5, 11641. [PubMed: 26119007]

(45). Ren ZJ; Umble AK Recover wastewater resources locally. Nature 2016, 529 (7584), 25-25. 


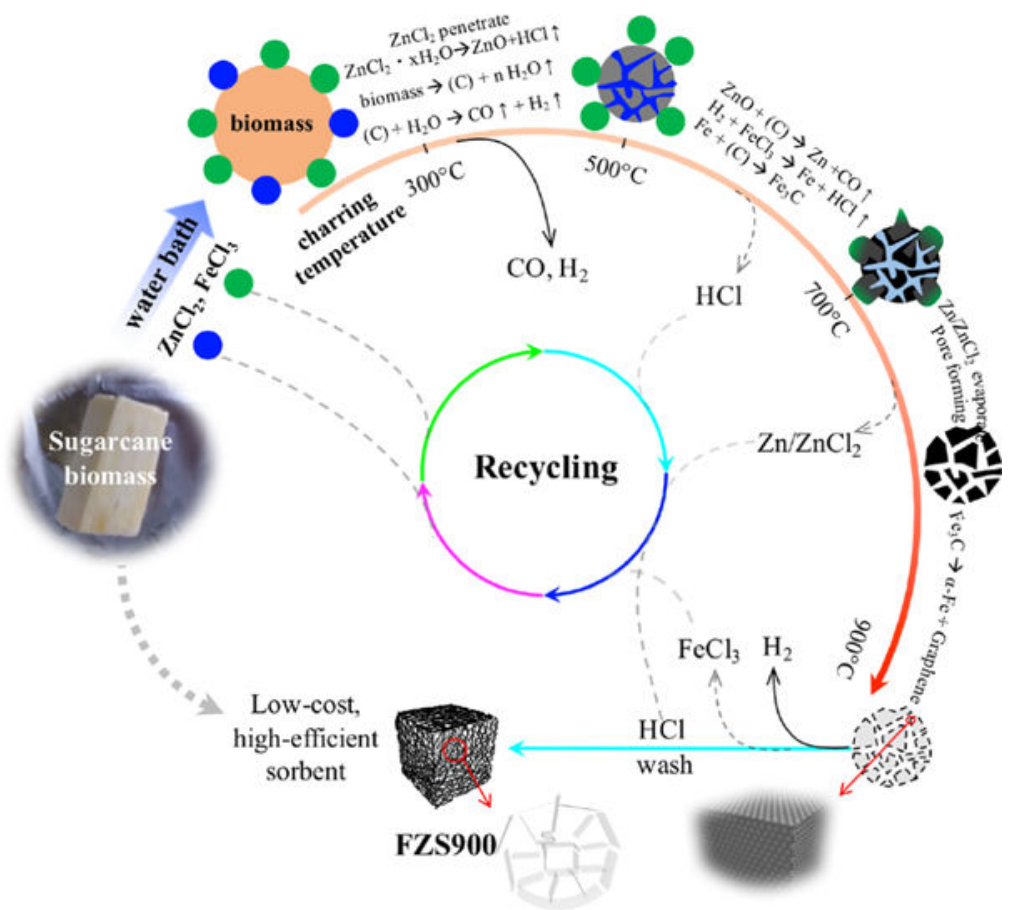

Figure 1.

Preparation of the graphene-like FZS900 material derived from sugar cane biomass and the possible reaction route. 

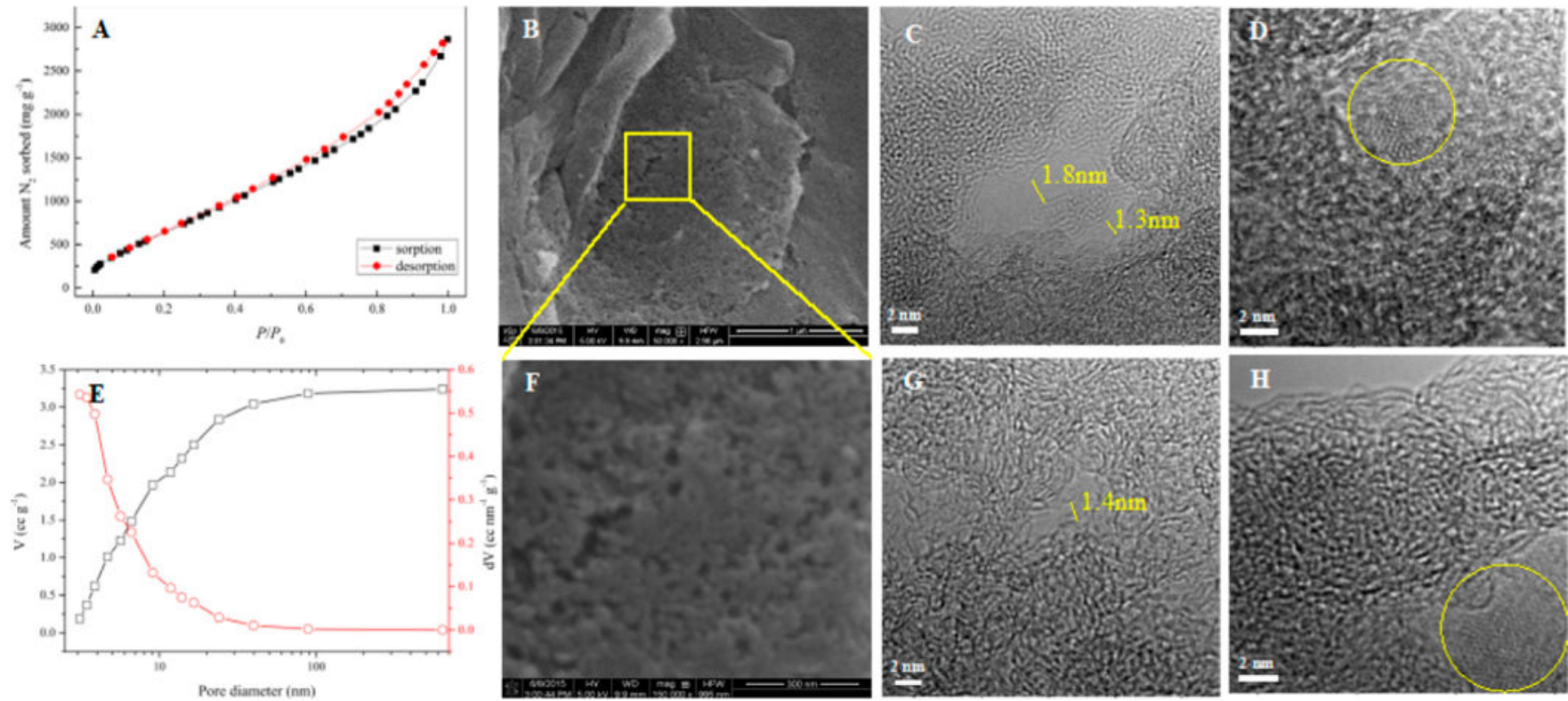

Figure 2.

(A) $\mathrm{N}_{2}$ adsorption-desorption isotherms of FZS900 at $77 \mathrm{~K}$ during BET- $\mathrm{N}_{2}$ characterization and (E) the corresponding pore size distribution calculated from the $\mathrm{N}_{2}$-desorption data in (A) according to the Barrett-Joyner-Halenda (BJH) method. (B, F) SEM and (C, G) HRTEM images of the microstructures and pore size of FZS900. (D, H) HRTEM images of the graphene-like nanosheet structure. The scale bars for (B) and (F) are $1 \mu \mathrm{m}$ and $300 \mathrm{~nm}$. 

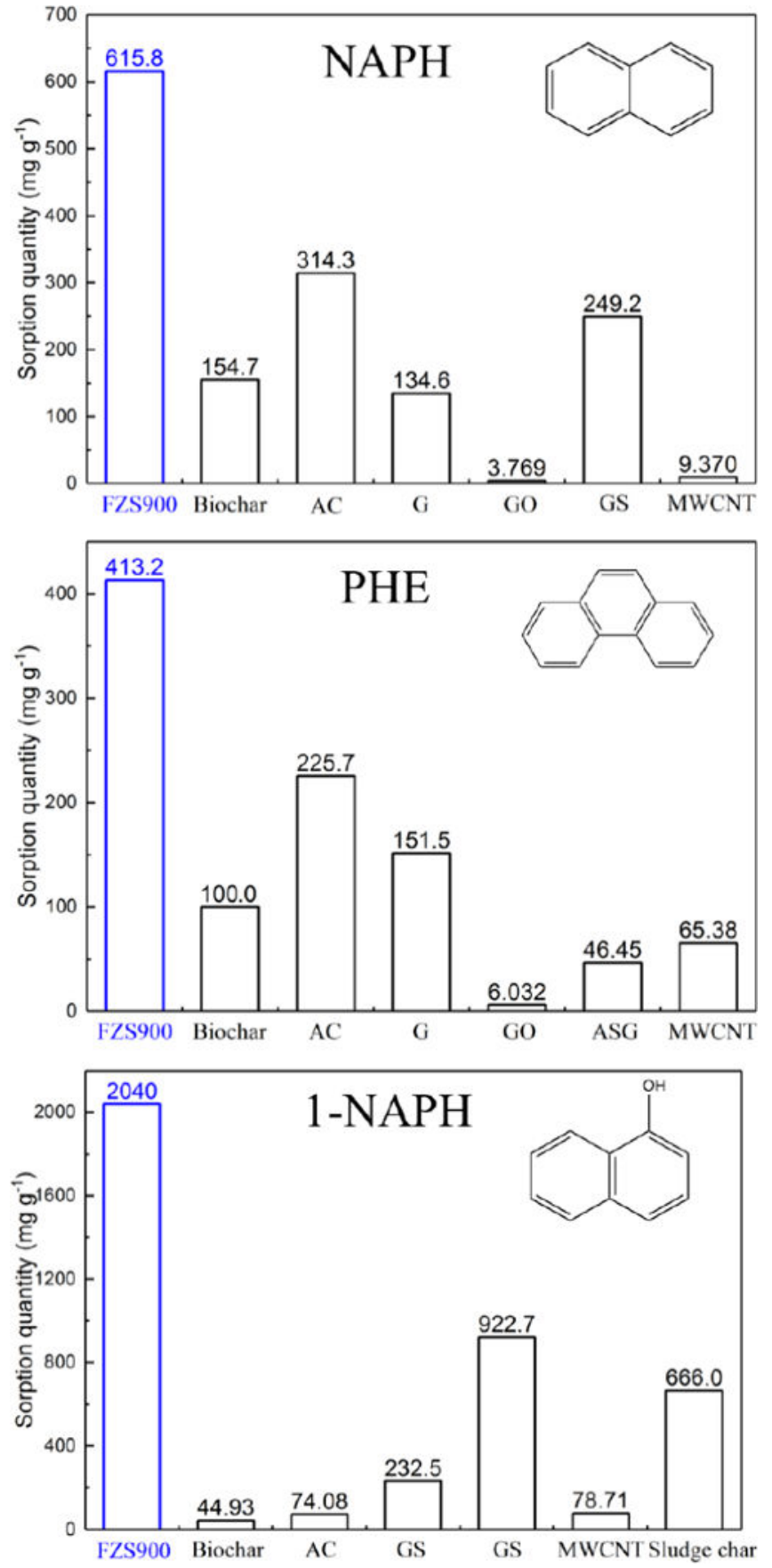

Figure 3.

Comparison of the adsorption data of FZS900 (blue columns) in the current study with that of traditional and novel materials from the literature. The sorption data for NAPH were obtained at $\mathrm{C}_{\mathrm{e}}=16.44 \mathrm{mg} \mathrm{L}^{-1}$, for PHE at $\mathrm{C}_{\mathrm{e}}=0.51 \mathrm{mg} \mathrm{L}^{-1}$, and for 1-NAPH at $\mathrm{C}_{\mathrm{e}}=103.0$ mg L ${ }^{-1}$. AC: activated carbon; G: graphene; GO: graphene oxide; GS: sulfonated graphene; MWCNT: multiwalled carbon nanotubes; ASG: aminosilica-supported graphene. Detailed data are presented in Table S4. 

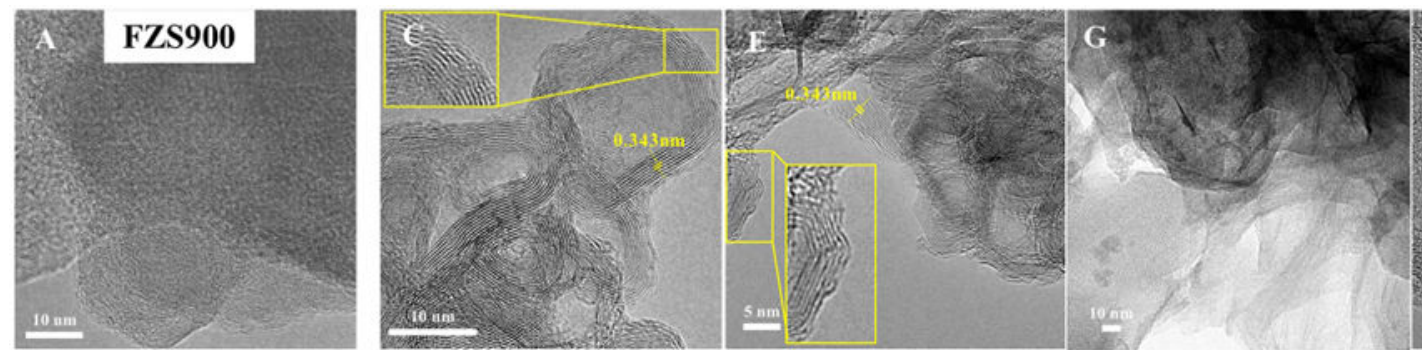

\section{1}

Proposed models before and after sorption
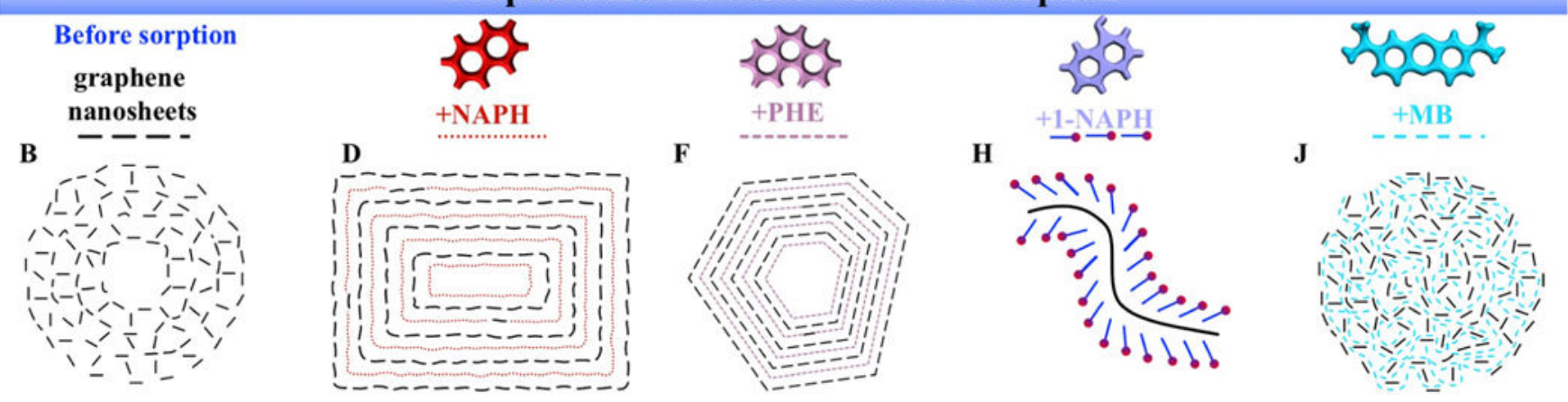

Figure 4.

HRTEM images of FZS900 (A) before and after (C) NAPH, (E) PHE, (G) 1-NAPH, and (I)

MB were adsorbed. Lower magnification images are given in Figure S2. (B) The proposed graphene nanosheet structure in FZS900. (D, F, H, J) The proposed model structure of FZS900 with adsorbed NAPH, PHE, 1-NAPH, and MB, respectively. 

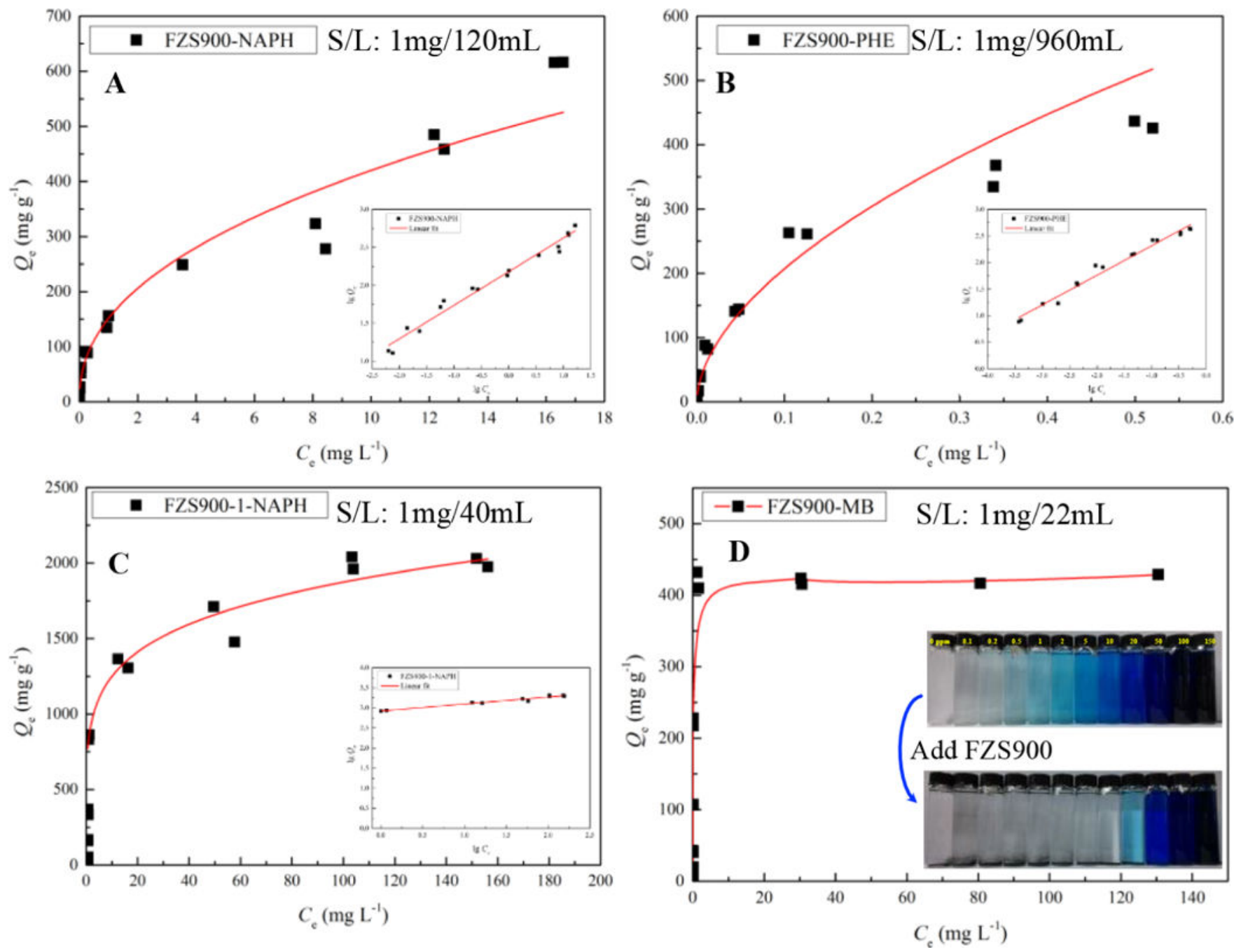

Figure 5.

Sorption isotherms of (A) NAPH, (B) PHE, (C) 1-NAPH, and (D) MB to FZS900. "S/L" at the top right corner of each figure refers to the solid-to-liquid ratio used for the corresponding compound. The $x$-axis refers to the equilibrium sorbate concentration $\left(\mathrm{C}_{\mathrm{e}}, \mathrm{mg}\right.$ $\left.\mathrm{L}^{-1}\right)$ in the aqueous phase, and the $y$-axis refers to the equilibrium sorbed quantity $\left(\mathrm{Q}_{\mathrm{e}}, \mathrm{mg} \mathrm{g}\right.$ ${ }^{-1}$ ) in the solid phase. In the bottom right corner of (A), (B), and (C) is the logarithmic axis of the sorption isotherm curves, which are fitted by the linear form of the Freundlich equation ( $\left.\lg \mathrm{Q}_{\mathrm{e}}=N \times \lg \mathrm{C}_{\mathrm{e}}+\lg K_{\mathrm{f}}\right)$. More information can be found in eqs $\mathrm{S} 3$ and $\mathrm{S} 4$, and the fitting results are given in Table S5. Kinetics results of NAPH and PHE sorption are presented in Figure S11. Partition coefficients $\left(K_{\mathrm{d}}, \mathrm{L} \mathrm{kg}^{-1}\right)$ are given in Figure S12. 